\title{
Study of Heart Rate Variability and Stress Markers in Basketball Players Submitted to Selective Loads Periodization System
}

\author{
José Henrique Mazon ${ }^{1}$, Ada Clarice Gastaldi ${ }^{1}$, Marli Cardoso Martins-Pinge ${ }^{2}$, \\ João Eduardo de Araújo ${ }^{1}$, Hugo Celso Dutra de Souza ${ }^{1, *}$ \\ ${ }^{1}$ Department of Rehabilitation and Functional Performance, University of São Paulo, Ribeirão Preto, Brazil \\ ${ }^{2}$ Department of Physiological Sciences, Londrina State University, Londrina, Brazil
}

\section{Email address:}

jhmazon@usp.br (J. H. Mazon), ada@fmrp.usp.br (A. C. Gastaldi), martinspinge@uel.br (M. C. Martins-Pinge), araujoje@fmrp.usp.br (J. E. de Araújo), hugocds@fmrp.usp.br (H. C. D. de Souza)

\section{To cite this article:}

José Henrique Mazon, Ada Clarice Gastaldi, Marli Cardoso Martins-Pinge, João Eduardo de Araújo, Hugo Celso Dutra de Souza. Study of Heart Rate Variability and Stress Markers in Basketball Players Submitted to Selective Loads Periodization System. American Journal of Sports Science. Vol. 3, No. 3, 2015, pp. 46-51. doi: 10.11648/j.ajss.20150303.12

\begin{abstract}
The decrease in the performance of athlete is often associated with an imbalance between workload and recovery period. Thus, it is very important to implement tools which can assist in the quantifying the effects of workloads, so that the maximum performance of the athlete is reached. In this context, we know little about the influence of selective load periodization system (SLPS) on cardiac autonomic control and the effects on stress markers already known. Thus, the aim of this study was to investigate if the application of SLPS promoted alterations in autonomic modulation of heart rate variability (HRV), as well as same stress markers. Therefore, sixteen male basketball players (mean \pm SE: age $23.3 \pm 1.0$ years; mass 87.5 $\pm 3.5 \mathrm{~kg}$; height $194 \pm 2 \mathrm{~cm}$ ) were submitted to SLPS and evaluated before and after a competition period. The HRV was evaluated by a spectral analysis of the time series composed of R-R intervals obtained in the supine position and during a tilt test. The evaluation of stress markers consisted of measuring plasma catecholamines, cortisol and free testosterone. The results demonstrated that the training load used during the competition period did not cause significant changes in the autonomic modulation of HRV. This affirmation is supported by the absence of change in oscillations of low frequency (LF: $0,04-0,15 \mathrm{~Hz}$ ), that corresponding to sympathetic and parasympathetic modulations, and high frequency (HF:0,15-05Hz), that corresponding only to parasympathetic modulations of HRV. Additionally, no changes were observed in plasma concentrations of catecholamines, free testosterone, cortisol and, consequently, in testosterone/cortisol ratio, when pre-competition and postcompetition values were compared. In summary, our findings suggest that the use of SLPS in basketball athletes presented balance between workloads and recovery periods. However, further investigations are needed, including in other sports, so that we can evaluate the effects of SLPS on cardiac autonomic modulation and stress markers evaluated in this study.
\end{abstract}

Keywords: Cardiac Autonomic Modulation, Catecholamines, Testosterone/Cortisol Ratio

\section{Introduction}

To improve performance in sports, athletes are subjected to intense training loads. Accordingly, to enable athletes to achieve a maximal performance at a desired time, it is necessary to strictly control their training loads through structuring and planning in periods or stages, also known as periodization [1]. Furthermore, thorough knowledge of the organic adaptive processes triggered in athletes by training is important [2]. Different methods of structuring or planning training workloads (periodization models or systems) are found in the literature. The classic or traditional model is characterised by three well-defined training periods: preparatory, competitive and transition $[3,4]$. The traditional periodization model has shown limitations in the training structure for high-performance athletes, especially collective modalities of sport. The increased frequency of competitions, globalisation, the fight against illegal drug use and advances in sports technology have caused changes in training patterns and prompted the use of alternative periodization models, called contemporary models [5]. These models, including the 
selective load periodization system (SLPS), are designed to fit the schedules of team sports for which the preparation periods before the start of competition are insufficient [6]. In SLPS, the training volume is maintained, and there is an increase in specific training tasks with a progressive expansion in the use of resources and methods directed at improving speed skills [2,6]. However, understanding the organic adaptations arising from the increase in specific training tasks that characterises contemporary periodization models remains a challenge for professionals working with high-performance athletes.

Therefore, it has been suggested that the evaluation and monitoring of stress markers during training may allow remodelling and individualisation of the workload to obtain maximal performance from athletes [7, 8]. Some studies have shown that the analysis of heart rate variability (HRV), indirectly quantifying the influence of autonomic, sympathetic and parasympathetic components on the heart, may be an important tool in the prognosis of stress and cardiovascular fitness in athletes [9, 10]. Additionally, studies are increasingly addressing the monitoring of hormonal responses to observe and control training-induced stress. There is a consensus that certain hormones, as free testosterone and cortisol, are primarily involved in the adaptive processes triggered by training loads or competition, in addition to influencing the regeneration phase $[8,11,12$, $13,14]$. However, the results are not conclusive with regard to stress-assessment models as methods to monitor the inherent adaptive training processes. Furthermore, in most cases, the systematization between workloads and the recovery period were determined empirically. Accordingly, studies on different systems of periodization, as well as distinct markers of physical stress, could collaborate with the systematic of the training, helping to control the individualization of workloads. Given the above, the purpose of this study was to investigate the effect of SLPS on known stress markers in basketball players before and after a competition period using the following approaches: 1) the spectral analysis of HRV using the autoregressive method and 2) the measurement of plasma catecholamines, cortisol and free testosterone. In this sense, our hypothesis was that the training organization by SLPS could minimize the load of physical stress imposed upon the athletes during the competition and that this response could be observed by monitoring of the HRV and hormonal stress markers evaluated in this study.

\section{Methods}

\subsection{Participants and Procedure}

The study was conducted with 16 male basketball players of high-performance (mean \pm SE: $23.3 \pm 1.0$ years; $87.5 \pm 3.5$ $\mathrm{kg} ; 1.94 \pm 0.02 \mathrm{~m}$ ) with training history of at least three years and submitted to the SLPS. The exclusion criteria adopted were the following: musculoskeletal changes that would prevent the athlete from performing the evaluations or participating in training and competitions and the use of drugs or substances that would interfere with cardiac autonomic function. The participants were screened in the Exercise Physiology Laboratory, Department of Rehabilitation and Functional Performance, Medical School of Ribeirão Preto, University of São Paulo, Brazil. The study was performed in accordance with the principles of the Declaration of Helsinki and the procedures adopted were approved by the Human Research Ethics Committee of the Clinical Hospital of the Medical School of Ribeirão Preto, University of São Paulo, Brazil (ethics approval n ${ }^{\circ}$ 62.079). All participants signed an informed consent form.

Table 1. Competition microcycle structure.

\begin{tabular}{|c|c|c|c|c|c|c|c|c|c|c|}
\hline Microcycles & 01 & 02 & 03 & 04 & 05 & 06 & 07 & 08 & 09 & 10 \\
\hline Total days of training & 6 & 7 & 6 & 6 & 7 & 6 & 7 & 6 & 7 & 6 \\
\hline No. of sessions & 12 & 13 & 11 & 12 & 12 & 12 & 13 & 11 & 12 & 12 \\
\hline Training time (min) & 920 & 960 & 900 & 920 & 900 & 920 & 960 & 900 & 900 & 920 \\
\hline Official games & 1 & 1 & 2 & 1 & 2 & 1 & 1 & 2 & 2 & 1 \\
\hline \multicolumn{11}{|c|}{ Motor skills and weekly frequency of use of training resources } \\
\hline \multicolumn{11}{|c|}{ Resistance } \\
\hline Technical and Tactical Work & 7 & 8 & 7 & 7 & 7 & 7 & 8 & 7 & 7 & 7 \\
\hline \multicolumn{11}{|l|}{ Strength } \\
\hline Weight-training exercises & 2 & 2 & 2 & 2 & 2 & 2 & 2 & 2 & 2 & 2 \\
\hline Resistance running & 1 & 1 & 1 & --- & 1 & -- & 1 & -- & 1 & --- \\
\hline Multi-jumps & --- & -- & -- & -- & --- & --- & -- & - & -- & -- \\
\hline Jumps with weight & -- & -- & -- & 1 & 1 & 1 & 1 & -- & -- & -- \\
\hline Depth jumps & 1 & 1 & -- & -- & -- & -- & -- & -- & --- & --- \\
\hline \multicolumn{11}{|l|}{ Speed } \\
\hline Maximum accelerations & --- & --- & 1 & 1 & 1 & 1 & 1 & 1 & 2 & 2 \\
\hline \multicolumn{11}{|l|}{ Flexibility } \\
\hline Dynamic stretching & 1 & 1 & -- & 1 & -- & 1 & --- & 1 & -- & 1 \\
\hline
\end{tabular}

The evaluations were conducted in two sessions: one before and one after a competition period. The competition period lasted a total of 10 weeks. Its purpose, as proposed by the SLPS, was the enhancement of the neuromuscular system 
with the increasing use of resources and methods to improve strength and speed skills. The microcycle composition of the competition period is described in Table 1.

\subsection{Blood Samples}

Blood samples were collected before and after the competition period between 7 and 8 hours using BD Vacutainer ${ }^{\circledR}$ EDTA tubes (Becton, Dickinson and Company, Franklin Lakes, NJ, USA). Occasionally, blood samples were also collected at 16 hours for cortisol quantification. The samples were centrifuged for 10 minutes at 3,000 rpm and $4^{\circ} \mathrm{C}$. The plasma isolated from each athlete was stored in Eppendorf tubes $(1.5 \mathrm{ml})$ in a freezer $\left(-80^{\circ} \mathrm{C}\right)$ for later analysis. A 48-hours interval was established between the last training session and blood collection in the laboratory. The athletes were instructed to fast for 12 hours and to avoid physical activity and the consumption of alcoholic and caffeinated beverages during this period. To quantify the plasma cortisol and free testosterone, specific radioimmunoassay procedures were used (Siemens Medical Diagnostics, Los Angeles, CA, USA). The quantification of plasma catecholamines was performed by high-performance liquid chromatography (HPLC) using a multichannel electrochemical detector (CoulArray 5600; ESA, Inc., Chelmsford, MA, USA).

\subsection{Heart Rate Variability}

To spectral analysis of HRV the athletes were instructed not to consume alcoholic and caffeinated beverages, to avoid physical activity and to maintain their usual diet for the 48 hours prior to testing. Heart rate assessment for the spectral analysis of HRV was conducted between 9 and 10 hours according to the following protocol: the athletes were placed in the supine position for 20 minutes on an electric orthostatic table. They were then placed passively in a tilted position at $75^{\circ}$ for another 20 minutes (tilt test). The assessment of heart rate (HR) in the supine position and during the tilt test was performed using an electrocardiogram (PowerLab ML866, ADInstruments, Bella Vista, NSW, Australia) with the respiratory rate set at 15 cycles/minute with the aid of a metronome. The time series were obtained from the adjacent RR intervals (RRi) and then divided into segments of 200 heartbeats that were superimposed over the segments of 100 heartbeats from the previous series. After calculating the mean and variance of each segment, the spectral analysis was performed using the autoregressive model [15-17]. The oscillatory components present in the stationary segments, heartbeat to heartbeat of the RRi, were calculated based on the Levinson-Durbin recursion according to Akaike's criteria [15]. This procedure allows an automatic quantification of the centre frequency and the influence of each relevant oscillatory component present in the interval series. The oscillatory components were classified as low frequency (LF) and high frequency (HF), showing oscillations in the frequency ranges of $0.04-0.15 \mathrm{~Hz}$ and 0.15 $0.5 \mathrm{~Hz}$, respectively. The effect of the LF and HF components on the variability of the RRi was expressed in normalised units obtained by calculating the percentage of LF and HF variability in consideration of the total force and after subtracting the very low-frequency (VLF) component (frequencies $<0.04 \mathrm{~Hz}$ ). The normalisation procedure tends to minimise the effect of changing the total force on the absolute values of the variability of the LF and HF components [15-17].

\subsection{Statistical Analysis}

The data were analysed by Sigma-Stat ${ }^{\circledR}$ program and are presented as mean and standard error. The results of the endogenous factors and the spectral analysis of HRV were analysed using the Student's t test for independent measures and the Mann-Whitney rank sum when necessary. The significance level was set at $\mathrm{p}$ less than $5 \%(\mathrm{p}<0.05)$ for all analyses.

\section{Results}
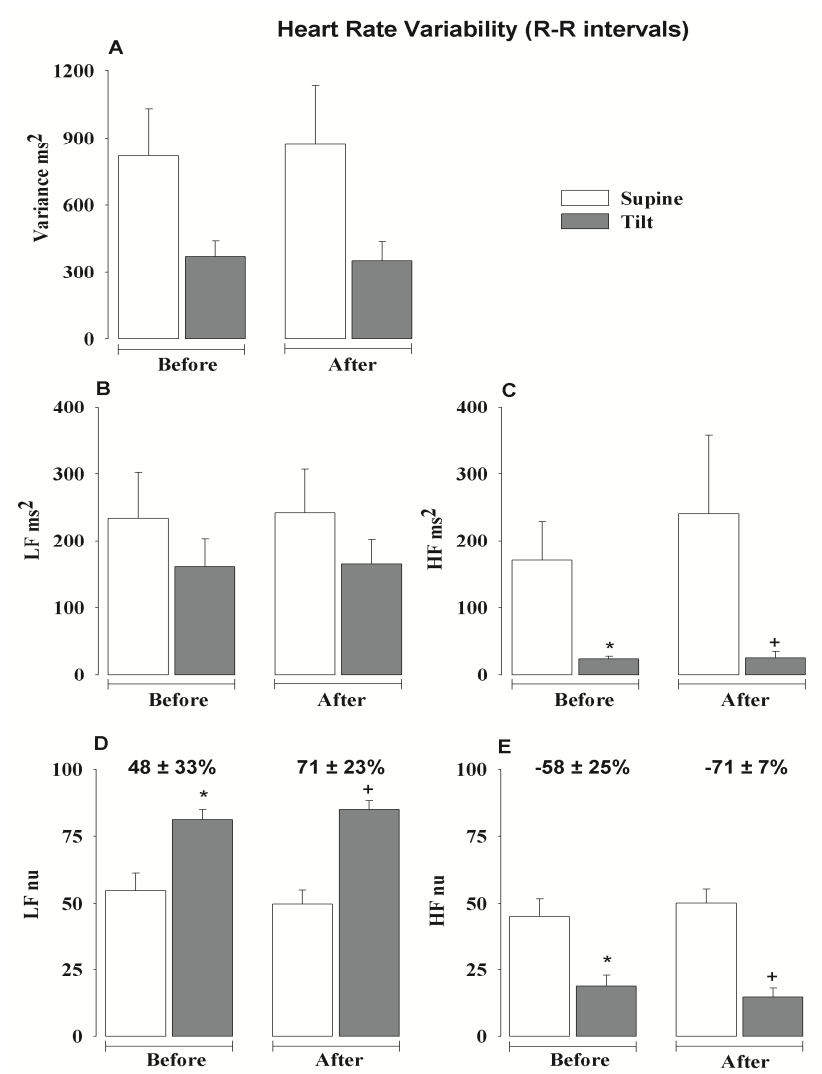

$-71 \pm 7 \%$

Figure 1. (A) Total variance of $R-R$ interval. Spectral power density of $R-R$ interval in low- $(L F)$ and high-frequency $(H F)$ bands in absolute $\left(\mathrm{ms}^{2} ; B\right.$ and C) and normalised units (nu; D and E) during the supine assessment and the tilt test (with their respective percentage change) calculated from time series in basketball players before and after the competition period. ${ }^{*} p<0.05 \mathrm{vs}$. the supine position before the competition period. ${ }^{+} p<0.05 \mathrm{vs}$. the supine position after the competition period.

The Figure 1 show the parameters of the spectral responses of HRV found in the supine position and during the tilt test in the athletes studied, before and after the competition period. In the supine assessment, the comparison between the two 
experimental periods showed no difference between the spectral parameters evaluated. The tilt test (inclination of $75^{\circ}$ from the supine position) produced similar results, which were characterised by an increase in the LF oscillations in normalised units (Figure 1D) and a reduction of the HF oscillations in absolute ( Figure 1C) and normalised units (Figure 1E). Similar values were observed in both experimental periods when comparing the percentage variation in relation to the normalised LF (Figure 1D) and HF (Figure 1E) oscillations after a tilt test.

The Table 2 depicts the hormone levels, comparing the values obtained before and after the competition period. In relation to plasma concentrations of catecholamines and free testosterone, no difference was observed between time points Similar results were observed in plasma cortisol levels. As shown in Table 2 and Figure 2, there was no difference in the testosterone/cortisol ratio before and after the competition period.

Table 2. Hormonal responses of basketball players before and after the competition period.

\begin{tabular}{lll}
\hline & Competition \\
\hline Hormonal Responses & Before & After \\
\hline Dopamine, $\mathrm{pg} / \mathrm{ml}$ & $30.0 \pm 2.9$ & $31.3 \pm 4.4$ \\
Adrenaline, $\mathrm{pg} / \mathrm{ml}$ & $43.3 \pm 6.5$ & $46.3 \pm 7.8$ \\
Noradrenaline, $\mathrm{pg} / \mathrm{ml}$ & $140.9 \pm 23.1$ & $177.2 \pm 24.6$ \\
Total catecholamines, $\mathrm{pg} / \mathrm{ml}$ & $184.2 \pm 26.1$ & $223.6 \pm 22.7$ \\
Cortisol, $\mu \mathrm{g} / 100 \mathrm{ml}$ & $13.8 \pm 1.2$ & $12.7 \pm 1.2$ \\
Free Testosterone, $\mathrm{pg} / \mathrm{ml}$ & $15.0 \pm 0.9$ & $16.5 \pm 1.6$ \\
Free Testosterone/Cortisol ratio & $1.18 \pm 0.1$ & $1.35 \pm 0.7$ \\
\hline
\end{tabular}

Values are expressed as means \pm S.E. ${ }^{*} p<0.05 v$ s. before the competition period.

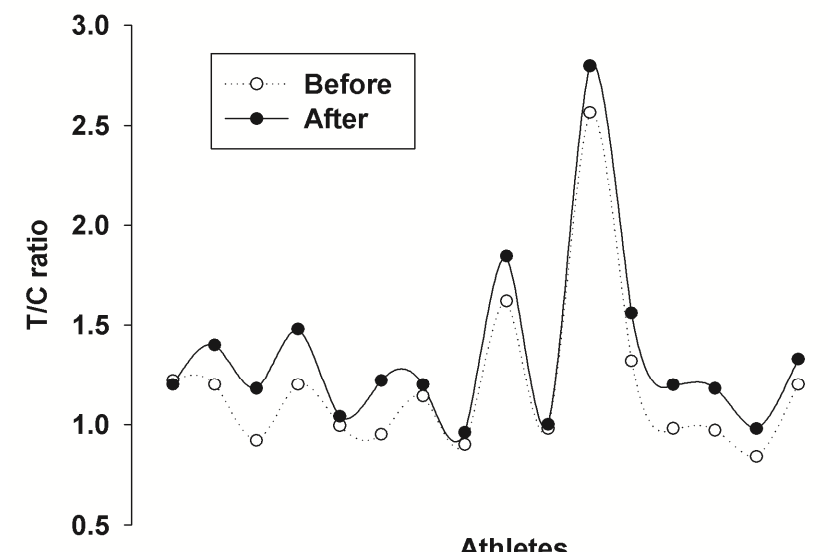

Figure 2. Individual values of testosterone/cortisol ratio in basketball players before and after the competition period.

\section{Discussion}

The plateauing of or decrease in the maximal performance of elite athletes is often associated with an imbalance between workload and recovery [18]. Accordingly, several studies have reported the monitoring of stress markers as a means to control the adaptations triggered by training or competition $[9,10,14]$.

In the present study, was investigated in basketball players undergoing SLPS training the change in the autonomic modulation of HRV and the plasma concentrations of catecholamines, cortisol and free testosterone before and after a competition period. The main finding was that workloads and training used during the competition did not cause significant changes in cardiac autonomic modulation and stress markers evaluated. This conclusion is supported by the absence of change in the HRV, plasma concentrations of catecholamines, free testosterone, cortisol and testosterone/cortisol ratio, when pre-competition and postcompetition values were compared.

It is well established in the literature that physical exercise improves cardiac autonomic modulation as compared with values obtained during the sedentary phase. This improvement is frequently associated with increased HRV, mainly because of the increased influence of the parasympathetic autonomic component on the heart [19-21]. However, the cardiac modulatory balance exerted by the sympathetic and parasympathetic autonomic components may be closely related to the type of physical exercise [21].

In the present study, was not observed any change in the autonomic modulation of HRV when comparing the two periods evaluated (before and after the competition period). These results may be related to a possible adaptation of athletes to the training intensity during this period, given that a decrease in this marker has been previously demonstrated in athletes with insufficient recovery between training sessions [10]. However, little is known about the relationship between training intensity and the adaptations involving cardiac autonomic modulation in athletes. Thus, it is possible that our findings are associated with the fact that basketball is an intermittent sport in which athletes often perform highintensity and short-duration work. This hypothesis corroborates the findings of other studies that showed that the cardiac autonomic control did not change in athletes subjected to intense training $[22,23]$. The precise cause of the results for autonomic modulation must be investigated. However, it is possible that autonomic modulation is associated with a cardiovascular preconditioning of these athletes, that is, an improvement in the modulation of the cardiac autonomic balance acquired before the start of the competition period. Another finding related to cardiac autonomic modulation was observed during the tilt test. Lowfrequency oscillations in absolute units were not altered in the two evaluated periods (before and after the competition period). This behaviour seems to have been influenced by the oscillations induced by parasympathetic autonomic modulation, given that the LF oscillations reflect the sum of the sympathetic and parasympathetic autonomic components $[17,24]$. In this case, the results suggest that the absence of changes in the LF oscillations in absolute units during the tilt test for the two periods are caused by a modulatory autonomic balance characterised by increased sympathetic 
modulatory influence and the removal of parasympathetic modulatory influence, resulting in no significant change in the LF oscillations in absolute values. This hypothesis seems to gain strength with the prominent reduction of $\mathrm{HF}$ oscillations observed during the tilt test. In contrast, in normalised units, the LF oscillations increased when the tilt test was performed because the normalised values represent the calculation of the percentage share of the LF and HF bands in total power after the subtraction of the very lowfrequency component (VLF $<0.04 \mathrm{~Hz})$. Consequently, because the HF oscillations decreased significantly during the $75^{\circ}$ inclination employed in the tilt test, the percentage share of LF oscillations significantly increased. These results were similar in both periods evaluated, as shown in Figure 1, depicting the numerical values of the percentage of the obtained responses.

As for the hormonal measurements, studies have shown that by monitoring the blood levels of catecholamines, cortisol and free testosterone, the level of physical stress imposed by workloads in a given training or competition period can be identified $[25,26]$. However, factors such as exercise characteristics (type, duration and intensity), training, age, emotional state and nutritional status can also influence the responses of these hormones [25], and the literature shows contradictory results regarding the effects of training on the responses of these hormones. With respect to catecholamines, a study involving volleyball athletes showed increased serum levels of adrenaline and reduced levels of noradrenaline during a competition period [27]. In contrast, other authors have shown that the training programme does not affect catecholamines concentrations [28, 29].

In the present study, no significant changes were observed in the plasma concentration of dopamine, adrenaline, noradrenaline and total catecholamines before and after the competition period. These results demonstrate a direct correlation with cardiac autonomic modulation, which did not change after the competition period. However, because catecholamines are regarded as hormonal stress markers [30, $31]$, the results seem to reflect positive adjustments to the workloads during competition, and a previous study reported an increase in the secretion of these hormones as a result of physiological and mental stress in high-performance athletes during a competition period [32].

In addition, free testosterone and cortisol showed interesting results. Testosterone is recognised as a hormone involved in musculoskeletal anabolic processes, whereas cortisol has catabolic functions [14]. In the present study, there was no change in the plasma concentration of free testosterone and cortisol after the competition period. The literature reports no consistent patterns for the traininginduced adaptations in the basal concentrations of these hormones. Therefore, the testosterone/cortisol ratio has been suggested as a potent endocrine biomarker of the anaboliccatabolic balance and a tool for diagnosing overreaching and overtraining in athletes $[13,33,34]$. In the present study, was not observed change in the testosterone/cortisol ratio, which seems to indicate the maintenance of the anabolic-catabolic balance of athletes after the competition period [35].

\section{Conclusions}

The results showed that the training loads used in this study, during the competition, did not cause significant changes in cardiac autonomic modulation and stress markers evaluated. This conclusion is supported by the absence of change in the HRV, plasma concentrations of catecholamines, free testosterone, cortisol and testosterone/cortisol ratio, when pre-competition and post-competition values were compared. However, further investigations are needed, including in other sports, so that we can evaluate the effects of SLPS on cardiac autonomic modulation and stress markers evaluated in this study.

\section{Acknowledgement}

The authors declare that they have no conflicts of interest concerning this article and are grateful to São Paulo Research Foundation (FAPESP-2012/04299-8) for the financial support.

\section{References}

[1] Bompa T, Haff G. Periodization: theory and methodology of training. Human Kinetics, Champaign, 2009.

[2] Moreira, A. La periodización del entrenamiento y las cuestiones emergentes: el caso de los deportes de equipo [Periodization of training and emerging issues: the case of team sports]. Rev Andal Med Deporte 2010; 3: 170-178.

[3] Matveev LP. Treino Desportivo: metodologia e planejamento. Guarulhos, SP: Phorte, 1997.

[4] Issurin, V. Block periodization versus traditional training theory: a review. J Sports Med Phys Fitness 2008; 48: 65-75.

[5] Issurin, V. New Horizons for the Methodology and Physiology of Training Periodization. Sports Med 2010; 40: 189-206.

[6] Gomes, AC. Treinamento desportivo - estruturação e periodização [Sports training - structuring and periodization]. 2rd ed., Porto Alegre, SC: Artmed, 2009.

[7] Hoffman JR, Kang J, Ratamess NA, Faigenbaum AD. Biochemical and hormonal responses during an intercollegiate football season. Med Sci Sports Exerc 2005; 37: 1237-1241.

[8] Schelling X, Calleja-González J, Torres-Ronda L, Terrados N. Using testosterone and cortisol as biomarker for training individualization in elite basketball: a 4-year follow-up study. J Strength Cond Res 2015; 29: 368-378.

[9] Kiviniemi AM, Hautala AJ, Kinnunem H, Tulppo MP. Endurance training guided individually by daily heart rate variability measurements. Eur J Appl Physiol 2007; 101: 743 751.

[10] Kiviniemi AM, Hautala AJ, Mäkikallio TH, Seppänen T, Huikuri HV, Tulppo MP. Cardiac vagal outflow after aerobic training by analysis of high-frequency oscillation of the R-R interval. Eur J Appl Physiol 2006; 96: 686-692. 
[11] Lehmann M, Gastmann U, Petersen KG, Bachl N, Seidel A, Khalaf AN, Fischer S, Keul J. Training - overtraining: performance, and hormone levels, after a defined increase in training volume versus intensity in experienced middle and long distance runners. Br J Sports Med 1992; 26: 233-242.

[12] Papacosta E, Nassis GP. Saliva as a tool for monitoring steroid peptide and immune markers in sport and exercise science. J Sci Med Sport 2011; 14: 424-434.

[13] Hug M, Mullis PE, Vogt M, Ventura N, Hoppeler H. Training modalities: over-reaching and over-training in athletes, including a study of the role of hormones. Best Pract Res Clin Endocrinol Metab 2003; 17: 191-209.

[14] Kraemer WJ, Ratamess NA. Hormonal responses and adaptations to resistance exercise and training. Sports Med 2005; 35: 339-361.

[15] Malliani A, Pagani M, Lombardi F, Cerutti S. Cardiovascular neural regulation explored in the frequency domain. Circulation 1991; 84: 482-492.

[16] Rubini R, Porta A, Baselli G, Cerutti S, Paro M. Power spectrum analysis of cardiovascular variability monitored by telemetry in conscious unrestrained rats. J Auton Nerv Syst 1993; 45: 181-190.

[17] Task Force. Heart rate variability: standards of measurement, physiological interpretation and clinical use. Task Force of the European Society of Cardiology and the North American Society of Pacing and Electrophysiology. Circulation 1996; 93: 1043-1065.

[18] Hartwig TB, Naughton G, Searl J. Load, stress, and recovery in adolescent rugby union players during a competitive season. J Sports Sci 2009; 27: 1087-1094.

[19] Raczak G, Daniłowicz-Szymanowicz L, KobuszewskaChwirot M, Ratkowski W, Figura-Chmielewska M, Szwoch M Long-term exercise training improves autonomic nervous system profile in professional runners. Kardio Pol 2006; 64: $135-140$.

[20] Sztajzel J, Jung M, Sievert K, Bayes De Luna A. Cardiac autonomic profile in different sports disciplines during all-day activity. J Sports Med Phys Fitness 2008; 48: 495-501.

[21] Collier SR, Kanaley JA, Carhart RJr, Frechette V, Tobin MM, Bennett N, Luckenbaugh AN, Fernhall B. Cardiac autonomic function and baroreflex changes following 4 weeks of resistance versus aerobic training in individuals with prehypertension. Acta Physiol 2009; 195: 339-348.

[22] Bonaduce D, Petretta M, Cavallaro V, Apicella C, Ianniciello A, Romano M, Breglio R, Marciano F. Intensive training and cardiac autonomic control in high level athletes. Med Sci Sports Exerc 1998; 30: 691-696.
[23] Vinet A, Beck L, Nottin S, Obert P. Effect of intensive training on heart rate variability in prepubertal swimmers. Eur J Clin Invest 2005; 35: 610-614.

[24] Souza HC, Martins-Pinge MC, Dias da Silva VJ, Borghi-Silva A, Gastaldi AC, Blanco JH, Tezini GC. Heart rate and arterial pressure variability in the experimental renovascular hypertension model in rats. Auton Neurosci 2008; 139: 38-45.

[25] Zouhal H, Jacob C, Delamarche P, Gratas-Delamarche A. Catecholamines and the effects of exercise, training and gender. Sports Med 2008; 38: 401-423.

[26] Lane AR, Duke JW, Hackney AC. Influence of dietary carbohydrate intake on the free testosterone: cortisol ratio responses to short-term intensive exercise training. Eur J Appl Physiol 2010; 108: 1125-1131.

[27] Mazon J, Gastaldi A, Di Sacco T, Cozza I, Dutra S, Souza H. Effects of training periodization on cardiac autonomic modulation and endogenous stress markers in volleyball players. Scand J Med Sci Sports 2013; 23: 114-120.

[28] Nevill ME, Boobis LH, Brooks S, Williams C. Effect of training on muscle metabolism during treadmill sprinting. $\mathrm{J}$ Appl Physiol 1989; 67: 2376-2382.

[29] Jacob C, Zouhal H, Vincent S, Gratas-Delamarche A, Berthon PM, Bentué-Ferrer D, Delamarche P. Training status (endurance or sprint) and catecholamine response to the Wingate-test in women. Int J Sports Med 2002; 23: 342-347.

[30] Frankenhaeuser M, Dunne E, Lundberg U. Sex differences in sympathetic-adrenal medullary reactions induced by different stressors. Psychopharmacology 1976; 47: 1-5.

[31] Zouhal H, Jacob C, Rannou F, Gratas-Delamarche A, BentuéFerrer D, Delamarche P. Effect of training status on the sympathoadrenal activity during a supramaximal exercise in human. J Sports Med Phys Fitness 2001; 41: 330-336.

[32] Garatachea N, Hernández-García R, Villaverde C, GonzálezGallego J, Torres-Luque G. Effects of 7-weeks competitive training period on physiological and mental condition of top level judoists. J Sports Med Phys Fitness 2012; 52: 1-10.

[33] Urhausen A, Gabriel H, Kindermann W. Blood hormones as markers of training stress and overtraining. Sports Med 1995; 20: $251-276$.

[34] Silva JR, Ascensão A, Marques F, Seabra A, Rebelo A, Magalhães J. Neuromuscular function, hormonal and redox status and muscle damage of professional soccer players after a high-level competitive match. Eur J Appl Physiol 2013; 113: 2193-2201.

[35] Lac G, Berthon P. Changes in cortisol and testosterone levels and $\mathrm{T} / \mathrm{C}$ ratio during an endurance competition and recovery. $\mathrm{J}$ Sports Med Phys Fitness 2000; 40: 139-144. 